\section{Utilidad de la prueba Aspergillus-LFD para el diagnóstico de aspergilosis: primera experiencia en Chile}

\author{
Eduardo Álvarez
}

\section{Utility of Aspergillus-LFD: first experience in Chile}

The diagnosis of invasive aspergillosis remains a challenge. Detection of galactomannan in serum and bronchoalveolar lavage is a useful tool; however due to methodological and economic reasons, the test frequencies of galactomannan assays vary from daily to weekly, which constitute a risk to the patient. In this study, we aimed to evaluate and correlate the performance of the new kit Aspergillus-LFD with the GMEIA. Aspergillus-LFD kit represents a fast, economical and simple test; showed a good performance and excellent correlation with GM-EIA kit. Given the above, the Aspergillus-LFD is emerging as an alternative to consider in the early diagnosis of invasive aspergillosis.

Key words: Aspergillosis, galactomannan, biomarker, lateral-flow device, invasive fungal diseases.

Palabras clave: Aspergillosis, galactomanano, biomarcadores, LFD, enfermedad fúngica invasora.

\section{Introducción}

La enfermedad fúngica invasora (EFI) es una entidad que se asocia con una morbi-mortalidad significativa ${ }^{1}$. Una de las EFI más prevalentes es la aspergilosis invasora (AI), causada principalmente por Aspergillus fumigatus. El diagnóstico temprano de la AI es crucial para el inicio de una terapia con resultados satisfactorios en pacientes de alto riesgo. Asimismo, la detección precisa y temprana reduce el uso innecesario de antifúngicos ${ }^{2-4}$. Según lo anterior, se hace necesario contar con herramientas de diagnóstico rápidas y eficaces ${ }^{5}$. Hasta ahora, el método de mejor rendimiento para el diagnóstico de EFI es la detección de antígenos fúngicos en el suero. Una de las pruebas ampliamente utilizadas para este propósito es Platelia ${ }^{\mathrm{TM}}$ Aspergillus EIA (GM-EIA), un inmunoensayo enzimático que detecta la presencia de galactomanano (GM), principal componente de la pared celular de Aspergillus. El kit GM-EIA ha demostrado un buen rendimiento en el diagnóstico de AI a partir de muestras de suero ${ }^{4,6}$; al utilizarse un índice de densidad óptica (IDO) de 0,5 como valor de corte. Se ha reportado una sensibilidad y especificidad aproximada de $78 \%$ y $81 \%$; respectivamente ${ }^{6}$. A pesar de que GM-EIA tiene un rendimiento diagnóstico aceptable, presenta

\footnotetext{
Universidad de Chile

Instituto de Ciencias Biomédicas

Unidad Micología - Hongos Filamentosos

Recibido: 15 de septiembre de 2014 / Aceptado: 30 de diciembre de 2014.
}

No hay conflicto de intereses

Financiamiento: Sin financiamiento externo.

Correspondencia a:

ealvarezd@med.uchile.cl
Tabla 1. Comparación del costo y material necesarios para los kit Aspergillus-LFD y GM-EIA

\begin{tabular}{|c|c|c|}
\hline & Aspergillus-LFD & GM-EIA \\
\hline Material requerido & + (pipeta, centrífuga) & $\begin{array}{l}+++ \text { (pipeta, centrífuga, } \\
\text { baño maría,lector placas, etc.) }\end{array}$ \\
\hline Costo & + $\$ 15.000$ por test apróx. & $\begin{array}{l}++\$ 25.000-\$ 45.000 \text { por } \\
\text { test apróx. }\end{array}$ \\
\hline Tiempo de trabajo & + 20 minutos apróx. & +++4 horas apróx. \\
\hline
\end{tabular}

algunos inconvenientes metodológicos, tales como el tiempo de ejecución $(3 \mathrm{~h})$, necesidad de instalaciones adecuadamente equipadas y costo económico. Dichos factores son los responsables de que la frecuencia de realización de la prueba GM-EIA pase de ser diaria a semanal, teniendo como consecuencia que los resultados pueden estar disponibles demasiado tarde para ser clínicamente útiles. Una alternativa útil para superar los inconvenientes antes citados es la utilización de pruebas basadas en inmunocromatografía (tecnología de flujo lateral o lateral flow device-LFD, en inglés). Recientemente ha sido desarrollada la prueba Aspergillus-LFD, que consiste en un ensayo inmunocromatográfico que detecta una glicoproteína extracelular de $A$. fumigatus en el suero o en lavado broncoalveolar (LBA). Entre sus ventajas destacan el tiempo de ejecución ( $15 \mathrm{~min}$ ), su simplicidad, y el bajo costo comparado con otras técnicas como GM o la detección de $\beta$-glucanos ${ }^{7}$ (Tabla 1). Estudios recientes, realizados con sueros obtenidos de modelos animales y con LBA de pacientes con neoplasias y sometidos a trasplante de órganos sólidos, han demostrado la utilidad de Aspergillus-LFD ${ }^{8-10}$.

El objetivo del estudio fue comparar el rendimiento diagnóstico del kit Aspergillus-LFD con la detección de GM por GM-EIA en una colección de sueros.

\section{Métodos}

Se evaluaron un total de 30 muestras del Laboratorio de Hongos Filamentosos de la Unidad de Micología de la Universidad de Chile: 25 muestras de suero y dos de LBA. Todas las muestras fueron sometidas previamente a la detección de GM con el kit GM-EIA. Además, se utilizó como control positivo una muestra de suero con resultado positivo para GM, y RPC-TR; como control negativo, un suero con resultado negativo para las pruebas de GM y RPC-TR, y como control de reacción cruzada, un suero con diagnóstico de fusariosis.

Para la determinación con el kit Aspergillus-LFD se procedió a mezclar $50 \mathrm{uL}$ de suero con $100 \mathrm{uL}$ de PBS / EDTA (4\% w/v) y se incubó durante 3 min a $100^{\circ} \mathrm{C}$. Tras una centrifugación por 5 min a $16.000 \mathrm{~g}$. se depositaron $100 \mathrm{uL}$ del sobrenadante en la zona de carga del kit Aspergillus-LFD. Los resultados fueron leídos después de 15 min (Figura 1).

Para los análisis estadísticos de sensibilidad y especificidad se utilizó el programa MedCalc $\AA 14$.

\section{Resultados}

Un total de 30 sueros fueron evaluados con Aspergillus-LFD (Tabla 2). Del total de muestras, con la prueba de detección de GM fue posible 


\begin{tabular}{|c|c|c|c|c|}
\hline SAMPLE & & ODI & GM & LFD \\
\hline 1 & Suero & 0,07 & Negativo & Negativo \\
\hline 2 & Suero & 0,7 & Positivo & Positivo \\
\hline 3 & Suero & 0,34 & Negativo & Negativo \\
\hline 4 & Suero & 0,37 & Negativo & Negativo \\
\hline 5 & Suero & 2,37 & Positivo & Positivo \\
\hline 6 & Suero & 0,8 & Positivo & Positivo \\
\hline 7 & LBA & 1,11 & Positivo & Positivo \\
\hline 8 & Suero & 0,67 & Positivo & Positivo \\
\hline 9 & Suero & 0,65 & Positivo & Positivo \\
\hline 10 & Suero & 0,58 & Positivo & Ligeramente positivo \\
\hline 11 & Suero & 1,49 & Positivo & Positivo \\
\hline 12 & Suero & 0,6 & Positivo & Positivo \\
\hline 13 & Suero & 1,5 & Positivo & Positivo \\
\hline 14 & Suero & 0,7 & Positivo & Positivo \\
\hline 22 & Suero & 2,37 & Positivo & Positivo \\
\hline 23 & Suero & 0,8 & Positivo & Positivo \\
\hline 34 & LBA & 1,11 & Positivo & Positivo \\
\hline 35 & Suero & 0,67 & Positivo & Positivo \\
\hline 36 & Suero & 0,65 & Positivo & Positivo \\
\hline 37 & Suero & 0,58 & Positivo & Positivo \\
\hline 38 & Suero & 1,49 & Positivo & Positivo \\
\hline 43 & Suero & 0,6 & Positivo & Positivo \\
\hline 44 & Suero & 1,5 & Positivo & Positivo \\
\hline 49 & Suero & 0,5 & Positivo & Positivo \\
\hline 51 & Suero & 0,51 & Positivo & Positivo \\
\hline 52 & Suero & 3,77 & Positivo & Positivo \\
\hline 53 & Suero & 1,69 & Positivo & Positivo \\
\hline Fus - 1 & Suero & 0,72 & Positivo & Negativo \\
\hline$C+$ & NA & 1,95 & Positivo & Positivo \\
\hline C - & NA & - & Negativo & Negativo \\
\hline
\end{tabular}

obtener un resultado positivo en 29 de ellas (sueros y LBA). Al evaluarse los sueros con Aspergillus-LFD se obtuvo una correlación de $100 \%$. No obstante, en una de las muestras evaluadas fue posible observar una débil reacción con Aspergillus-LFD. Dicha muestra obtuvo un índice de 0,58 en la prueba de GM. Según lo anterior, hubo una sensibilidad y especificidad de $100 \%$ para Aspergillus-LFD; superando a la especificidad mostrada por GM-EIA en el presente estudio (83\%).

Adicionalmente, y con el objetivo de evaluar una posible reacción cruzada con otros hongos filamentosos, se evaluó una muestra de suero

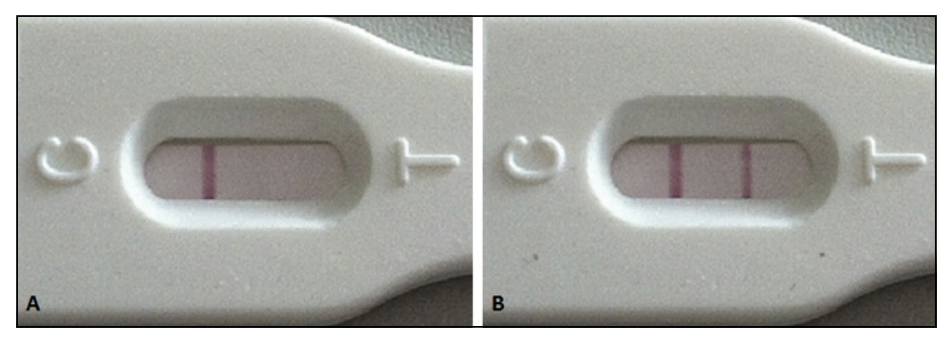

Figura 1. Visualización de las lineas testigo y muestra en el Aspergillus-LFD. (A) resultado negativo, (B) resultado positivo.

positiva para fusariosis; la cual obtuvo un resultado positivo (IDO $=0,72$ ) en la prueba de GM y negativo para Aspergillus-LFD.

Como control positivo se utilizó un suero con resultado positivo por GM y RPC-TR, obteniéndose una reacción positiva con Aspergillus-LFD. Con el control negativo no se obtuvo reacción en las pruebas de GM, RPC-TR, ni Aspergillus-LFD.

\section{Discusión}

El diagnóstico de la AI sigue siendo complejo, dado principalmente por lo inespecífico, costoso y laborioso de las técnicas actualmente utilizadas. Técnicas como la RPC-TR ofrecen una buena sensibilidad y especificidad; sin embargo, tiene desventajas como su estandarización y costosa implementación en laboratorios asistenciales; quedando limitadas casi exclusivamente a centros de investigación, al menos en nuestro medio.

Las técnicas rápidas y de bajo costo representan una ayuda real en el diagnóstico de EFI. Así, la inmunocromatografía conocida como LFD ha demostrado un buen rendimiento en el diagnóstico de criptococosis, y ahora, en el diagnóstico de AI. En este sentido, al realizar un análisis de los costos aproximados comparando Aspergillus- LFD y GM-EIA, el primero aventaja con creces al antiguo inmunoensayo enzimático en cuanto a tiempo de trabajo, equipamiento necesario y costos asociados; siendo éstos de casi un tercio (Tabla 1).

En un estudio en que se evaluaron 529 muestras de sueros, se comunicó que Aspergillus-LFD presentó una especificidad de 98\%, similar a la RPC $(96,6 \%)$ y levemente superior a GM-EIA $(91,5 \%)$. En cuanto a la sensibilidad, Aspergillus-LFD presentó un 81,8\%; siendo inferior que la RPC $(95,5 \%)$, pero mejor que GM-EIA $(77,3 \%)^{11}$. Nuestros resultados corroboran lo demostrado anteriormente, demostrando la mayor especificidad de Aspergillus-LFD (100\%) sobre GM-EIA (83\%); discriminando la presencia de antígenos de Fusarium de los de Aspergillus en la muestra de reacción cruzada. La sensibilidad para ambos métodos fue de $100 \%$ en el presente estudio.

En términos generales, podemos concluir la ventaja que significa el uso de Aspergillus-LFD en el diagnóstico de la AI con respecto a GM-EIA. La inmunocromatografía aquí ensayada, demostró ser altamente sensible y específica en sueros y LBA de pacientes con AI; superando a la detección de GM, permitiendo diferenciar una fusariosis de una aspergilosis. Asimismo, Aspergillus-LFD es una técnica fácil y rápida, pudiendo ser realizada por personal de laboratorio sin entrenamiento específico. La duración del método es de sólo $20 \mathrm{~min}$, contrastando con los 240 min necesarios para GM-EIA. Otro factor a destacar, es el bajo costo del nuevo LFD, representando 
casi un tercio del valor de una determinación de GM. Además, requiere una escasa implementación de equipos. Dado lo anteriomente expuesto, Aspergillus-LFD se perfila como la prueba de diagnóstico de AI de rutina para cualquier laboratorio de nuestro país, no siendo necesario invertir en entrenamiento de personal, equipos ni tiempo de trabajo.

A pesar del excelente rendimiento de Aspergillus-LFD demostrado, son necesarios nuevos estudios para evaluar un mayor número de muestras y así determinar con mayor certeza los resultados y aplicabilidad de la prueba en el diagnóstico temprano de la AI.

Agradecimientos: A OLM y G. McGonnell por la distribución del kit Aspergillus-LFD. A las Dras. Estibaliz Mateo y Valentina Salas por la revisión del manuscrito.

\section{Referencias bibliográficas}

1.- Kurosawa M, Yonezumi M, Hashino S, Tanaka J, Nishio M, Kaneda M, et al. Epidemiology and treatment outcome of invasive fungal infections in patients with hematological malignancies. Int J Hematol 2012; 96: 748-57.

2.- Maertens J, Theunissen K, Verhoef G, Verschakelen J, Lagrou K, Verbeken E, et al. Galactomannan and computed tomography-based preemptive antifungal therapy in neutropenic patients at high risk for invasive fungal infection: a prospective feasibility study. Clin Infect Dis 2005; 41: 1242-50.

3.- Odabasi Z, Mattiuzzi G, Estey E, Kantarjian H, Saeki F, Ridge R J, et al. Beta-D-glucan as a diagnostic adjunct for invasive fungal infections: validation, cutoff development, and performance in patients with acute myelogenous leukemia and myelodysplastic syndrome. Clin Infect Dis 2004; 39: 199-205.
4.- Pfeiffer C D, Fine J P, Safdar N. Diagnosis of invasive aspergillosis using a galactomannan assay: a meta-analysis. Clin Infect Dis 2006; 42: 1417-27.

5.- $\quad$ von Eiff M, Roos N, Schulten R, Hesse M, Zuhlsdorf M, van de Loo J. Pulmonary aspergillosis: early diagnosis improves survival. Respiration 1995; 62:341-7.

6.- Leeflang M M, Debets-Ossenkopp Y J, Visser C E, Scholten R J, Hooft L, Bijlmer H A, et al. Galactomannan detection for invasive aspergillosis in immunocompromised patients. Cochrane Database Syst Rev. 2008; 4:CD007394.

7.- Thornton C R. Development of an immunochromatographic lateral-flow device for rapid serodiagnosis of invasive aspergillosis. Clin Vaccine Immunol 2008; 15:1095-105.

8.- Wiederhold N P, Thornton C R, Najvar L K, Kirkpatrick W R, Bocanegra R, Patterson TF. Comparison of lateral flow technology and galactomannan and (1-3)-betaD-glucan assays for detection of invasive pulmonary aspergillosis. Clin Vaccine Immunol 2009; 16:1844-6.

9.- Wiederhold N P, Najvar L K, Bocanegra R, Kirkpatrick W R, Patterson T F, Thornton C R. Interlaboratory and interstudy reproducibility of a novel lateral-flow device and influence of antifungal therapy on detection of invasive pulmonary aspergillosis. J Clin Microbiol 2013; 51: 459-65.

10.- Hoenigl M, Koidl C, Duettmann W, Seeber K, Wagner J, Buzina W, et al. Bronchoalveolar lavage lateral-flow device test for invasive pulmonary aspergillosis diagnosis in haematological malignancy and solid organ transplant patients. J Infect 2012; 65: 588-91.

11.- White P L, Parr C, Thornton C, Barnes R A. Evaluation of real-time PCR, galactomannan enzyme-linked immunosorbent assay (ELISA), and a novel lateral-flow device for diagnosis of invasive aspergillosis. J Clin Microbiol 2013; 51: 1510-6. 